\title{
Executive dysfunction predicts treatment response to neuroplasticity-based computerized cognitive remediation (nCCR-GD) in elderly patients with major depression
}

\author{
Sarah Shizuko Morimoto, Psy.D. ${ }^{1,{ }^{*}}$, Faith M. Gunning, Ph.D. ${ }^{1}$, Bruce E. Wexler, M.D. ${ }^{2}$, Willie \\ Hu, B.S. ${ }^{1}$, Irena llieva, Ph.D. ${ }^{1}$, Jiacheng Liu, MD, PhD. ${ }^{3}$, Juliana Nitis ${ }^{1}$, and George S. \\ Alexopoulos, M.D. ${ }^{1}$ \\ ${ }^{1}$ Weill Cornell Medical College, Institute of Geriatric Psychiatry \\ ${ }^{2}$ Yale University Medical School, Department of Psychiatry \\ ${ }^{3}$ Zhongda Hospital, Medical School of Southeast University, Nanjing
}

\begin{abstract}
Objectives-Executive dysfunction (ED) is a predictor of poor treatment response of late-life depression to pharmacotherapy. In response to the consistency of these findings, we designed a neuroplasticity-based computerized cognitive remediation (nCCR-GD) intervention to target and improve ED in patients who failed to remit with antidepressant treatment. This study tests the hypothesis that ED at baseline will predict favorable treatment response to nCCR-GD.
\end{abstract}

Methods-11 elderly patients with treatment-resistant major depression were treated with a 30hour, 4-week, open, nCCR-GD treatment trial. Neuropsychological performance was assessed at baseline and after treatment ceased.

Results-ED at baseline was associated with greater reduction in Montgomery-Asberg Depression Rating Scale (MADRS) score over the 4-week treatment $\beta=-0.74, F(2,8)=10.85$, $p=0.009, R^{2}=0.55$.

Conclusions-ED predicts favorable treatment response to nCCR-GD in older adults suffering from major depression resistant to antidepressants. This finding is opposed to studies testing pharmacotherapy where ED predicts poorer treatment response.

\section{Keywords}

Computerized cognitive remediation; executive dysfunction; geriatric; depression; treatment resistant

\footnotetext{
*Corresponding Author: Sarah Shizuko Morimoto, Psy.D., Weill Cornell Medical College, 21 Bloomingdale Rd., White Plains, NY 10605, Phone: (914) 682-9100 ext. 5471, Fax: (914) 682-6979, ssm9006@med.cornell.edu.

No disclosures to report.

Publisher's Disclaimer: This is a PDF file of an unedited manuscript that has been accepted for publication. As a service to our customers we are providing this early version of the manuscript. The manuscript will undergo copyediting, typesetting, and review of the resulting proof before it is published in its final citable form. Please note that during the production process errors may be discovered which could affect the content, and all legal disclaimers that apply to the journal pertain.
} 


\section{Introduction}

Executive dysfunction is a predictor of poor treatment response of late-life depression to pharmacotherapy. Executive functions are cognitive control processes that modulate aspects of emotion and cognition, and are instantiated in the cognitive control network (CCN)[1]. The CCN is preferentially affected by aging, and structural, functional and behavioral evidence of impairment is commonly reported in geriatric depression [2,3]. Elderly depressed patients with these deficits are roughly two times less likely to reach remission than their unimpaired contemporaries[4, 5]. Even if their depressive symptoms subside, the currently available antidepressants (as a rule) do not improve executive dysfunction leaving patients perpetually disabled and susceptible to relapse[6].

Psychosocial treatments (e.g. Problem Solving Therapy (PST)) designed to ameliorate the impact of executive dysfunction on the patient and his/her environment have shown efficacy in treating depressive symptoms[7]. Depressed older adults with certain executive deficits respond better to PST than their cognitively normal peers. That is, contrary to clinical outcomes of pharmacotherapy, the more impaired patients' cognitive flexibility, the more they benefit from therapy [8]. However, similarly to antidepressants, while PST improves depression, it does not improve executive functions[9]. Taken together, these findings suggest that late-life depression is heterogeneous and may require treatments tailored to the individuals' particular cognitive profile. Further, for the almost $40 \%$ of older adults with depression and executive dysfunction, there is currently no treatment to address the totality of their illness.

The neuroscience of depression has progressed to a point where biologically meaningful targets can guide the design and evaluation of behavioral treatments. Recent analyses have focused on neurobehavioral predictors of treatment response in older adults with depression. These analyses are intended to isolate a target, or mediator of response to treatment, in order to understand the mechanisms of treatment response.

Based on findings implicating executive dysfunction in poor response to antidepressants, we developed a neuroplasticity-based computerized cognitive remediation (nCCR-GD) targeting executive dysfunction, and reported preliminary findings of efficacy [10]. nCCRGD targets performance on tasks related to the cognitive control network $(\mathrm{CCN})$ and assumes that neuroplasticity-related events can improve CCN function. Unlike nCCR-GD, PST provides skills to address everyday problems related to depressive symptoms and executive dysfunction rather than treating the underlying network dysfunction.

This study tests the hypothesis that deficits in executive functions in patients with geriatric depression will predict treatment response to nCCR-GD. We base this hypothesis on the assumption that resistance to antidepressants is contributed to by abnormal CCN function clinically expressed as executive dysfunction. 


\section{Methods}

\section{Participants}

Older adults (60-89) with major depression (by SCID-R/DSM-IV), who failed to achieve remission (MADRS>15) after treatment with therapeutic dosages of an selective serotonin reuptake inhibitor (SSRI) or selective serotonin/norepinephrine reuptake inhibitor (SNRI) antidepressant for at least 8 weeks. In addition, we asked that they and their physicians had no plan to change medication or dosages for the duration of the study (4 weeks) unless required by significant worsening of clinical symptoms. (For a detailed description of the sample please see Morimoto, Wexler et al 2014).

\section{nCCR-GD}

Participants completed 30 hours of cognitive remediation over 4 weeks on computer stations in private treatment rooms at the Weill Cornell Institute of Geriatric Psychiatry. The brief 4week time period was chosen to mitigate several factors: The selected participants were treatment resistant and quite symptomatic; nCCR-GD was an as-yet-untested treatment, and participants were asked to remain on their same (and ineffective) dose of medication. Participants had access to the supervising psychologist and research assistants for questions at any time, but after the initial program set up, participants worked on their own without intervention. (Please see Morimoto, Wexler et al., 2014.)

The Weill Cornell Institutional Review Board approved all procedures. After a complete description of the study to subjects, written informed consent was obtained.

\section{Outcome Measures}

Trained research assistants unaware of the study's hypotheses collected clinical ratings, neuropsychological tests and self-report measures under the supervision of a neuropsychologist (SSM).

Depressive symptoms were assessed using the 10-item MADRS. Disability was measured with the World Health Organization Disability Assessment Schedule-II (WHODAS-II). Baseline gross cognitive status was rated with the Mini-Mental State Examination.

Neuropsychological measures: Overall cognitive dysfunction was assessed with the Mattis Dementia Rating Scale (DRS). Executive functioning: cognitive flexibility with Trail Making Test B, semantic clustering with semantic clusters on the DRS Complex Verbal section of the Initiation/Perseveration (DRS I/P) subscale (Clusters of 3 or more words in a category, serially. If a perseveration occurred in the midst of a cluster, that cluster was not counted. Each cluster of three or more related words was given a score of "one" as in Troyer et. al, 1997)

Other neuropsychological domains tested included: Processing speed: with Trail Making Test A; verbal memory with the California Verbal Learning Test (CVLT-ii) long delay recall; and working memory with digits backwards from the Wechsler Adult Intelligence Scale (WAIS-IV). Alternate forms, where available, were used during the second administration. 


\section{Statistical Analysis}

To examine the relationship between executive functioning performance at baseline and change in symptoms of depression during the 4-week intervention, we performed a series of linear regressions with age entered as a covariate. To account for multiple comparisons we used the Bonferroni correction to lower the threshold of significance to 0.01 (.05/5). Our outcome was the change in participants' MADRS scores from baseline to week 4. To isolate the executive component of Trails B performance from processing speed, we subtracted the time to complete Trails A from time to complete Trails B (TB-TA)(in seconds). We then conducted a linear regression with the executive component of Trails B predicting MADRS difference scores from baseline to 4 weeks. We also tested the relationship of number of semantic clusters used to complete the complex verbal portion of the DRS I/P with change in MADRS score in the same manner. To test the relationship between cognitive domains other than targeted executive functions and change in symptoms of depression during the 4 week intervention, we tested the relationship of overall cognitive functioning (DRS); verbal memory (long delay recall scores on the CVLT-ii), processing speed (Trails A), and working memory (digits backwards, WAIS-IV) with MADRS difference scores with a linear regression.

\section{Results}

Higher TB-TA scores (indicating greater executive dysfunction) were associated with greater reduction in MADRS score over 4 weeks, $\beta=-0.74, F(2,8)=10.85, p=0.009, R^{2}=0.55$ (Figure 1.). We performed two posthoc analyses to clarify this relationship: Change in MADRS correlated with change in TB-TA over four weeks $\left(R^{2}=-0.50(\mathrm{p}=0.015)\right.$; baseline TB-TA predicted percent change of MADRS over the four weeks $\left(\mathrm{F}_{1,10}=5.20, \mathrm{p}=.049\right)$. No other cognitive variable at baseline was related to MADRS change over the intervention's 4 weeks including (CVLT long delay: $\beta=-0.04, F(2,8)=0.01, p=0.92, \mathrm{R}^{2}=0.001$; Digits Backwards: $\beta=0.56, F(2,8)=4.00, p=0.08, R^{2}=0.31$; Semantic Clustering: $\beta=0.20, \mathrm{~F}(2,8)=0.69, p=0.55$, $\left.R^{2}=0.04\right)$ Trails A: $\left.F(2,8)=0.09, p=.732, R^{2}=.022\right)$.

\section{Discussion}

The principal finding of this study is that deficits in executive functioning at baseline predict favorable treatment response to nCCR-GD in older adults suffering from major depression resistant to antidepressants. That is, patients who performed poorly on measures of cognitive flexibility exhibited greater reductions in their depressive symptoms than those with adequate performance. No other cognitive test performance predicted treatment response.

A potential interpretation of our findings is that nCCR-GD reduces depression by remediating deficits in executive functioning. A behavioral interpretation of these data may be that nCCR-driven executive benefits may promote a choice of effortful actions over habitual ones for the sake of more optimal long-term outcomes (e.g., stronger executive functioning may promote the conscious reframing of unrealistic negative thoughts, despite the automaticity of these thoughts). A potential neurobiological interpretation may be that increased activity and learning, induced by nCCR-GD, modifies CCN function, and thus improves a network abnormality contributing to poor response to antidepressants. These 
hypotheses open up avenues for future research, including mediation analyses and imaging studies, and provide the impetus for developing and testing combination therapies.

There are several important limitations that have variable impacts on the results of this preliminary study. The first is the study's small sample size. Given the small number of participants in this pilot trial, we can only speculate as to the generalizability of this finding. It also introduces several interpretational challenges, such as whether (or not) there is a meaningful relationship between improvement of depression and baseline Digit Span Backward (working memory performance). Second and perhaps most significant is the lack of an active, concurrently studied control group. A larger, randomized, double blind study is now underway. Last, the use of a combined intervention (nCCR-GD + antidepressant medication) does not allow us to distinguish if baseline executive dysfunction predicts response to $\mathrm{nCCR}-\mathrm{GD}$ alone and/or if nCCR-GD added to antidepressant medication is responsible for the reported results.

In conclusion, our findings, though preliminary, suggest that treatment resistant patients with baseline executive dysfunction respond more robustly to nCCR-GD than those patients without executive dysfunction. This finding is opposed to studies prescribing pharmacotherapy, where executive dysfunction predicts poorer treatment response. This finding, if replicated, has important theoretical and clinical implications about treatment mechanism, intervention development and selection.

\section{Acknowledgments}

This work was supported by National Institute of Mental Health grants P30 MH68638 (GSA), R01 MH079414 (GSA), T32 MH019132 (GSA), K23 MH095830 (SSM), UL1TR000457 (SSM) and the Sanchez Foundation.

\section{References}

1. Aizenstein HJ, et al. Altered functioning of the executive control circuit in late-life depression: episodic and persistent phenomena. Am J Geriatr Psychiatry. 2009; 17(1):30-42. [PubMed: 19001356]

2. Raz N, et al. Selective aging of the human cerebral cortex observed in vivo: differential vulnerability of the prefrontal gray matter. Cereb Cortex. 1997; 7(3):268-82. [PubMed: 9143446]

3. Gunning-Dixon FM, et al. Macromolecular white matter abnormalities in geriatric depression: a magnetization transfer imaging study. Am J Geriatr Psychiatry. 2008; 16(4):255-62. [PubMed: 18378551]

4. Morimoto SS, et al. Semantic organizational strategy predicts verbal memory and remission rate of geriatric depression. International journal of geriatric psychiatry. 2011

5. Morimoto SS, et al. Semantic organizational strategy predicts verbal memory and remission rate of geriatric depression. Int J Geriatr Psychiatry. 2012; 27(5):506-12. [PubMed: 21618287]

6 . Kiosses DN, et al. Executive dysfunction and disability in elderly patients with major depression. Am J Geriatr Psychiatry. 2001; 9(3):269-74. [PubMed: 11481135]

7. Arean PA, et al. Problem-solving therapy and supportive therapy in older adults with major depression and executive dysfunction. Am J Psychiatry. 2010; 167(11):1391-8. [PubMed: 20516155]

8. Beaudreau SA, et al. Does cognition predict treatment response and remission in psychotherapy for late-life depression? Am J Geriatr Psychiatry. 2015; 23(2):215-9. [PubMed: 25441055] 
9. Mackin RS, et al. Cognitive outcomes after psychotherapeutic interventions for major depression in older adults with executive dysfunction. Am J Geriatr Psychiatry. 2014; 22(12):1496-503. [PubMed: 24378255]

10. Morimoto SS, Wexler BE, Liu JC, Hu W, Seirup J, Alexopoulos GS. Neuroplasticity-based computerized cognitive remediation for treatment resistant geriatric depression. Nature Communications. 2014 


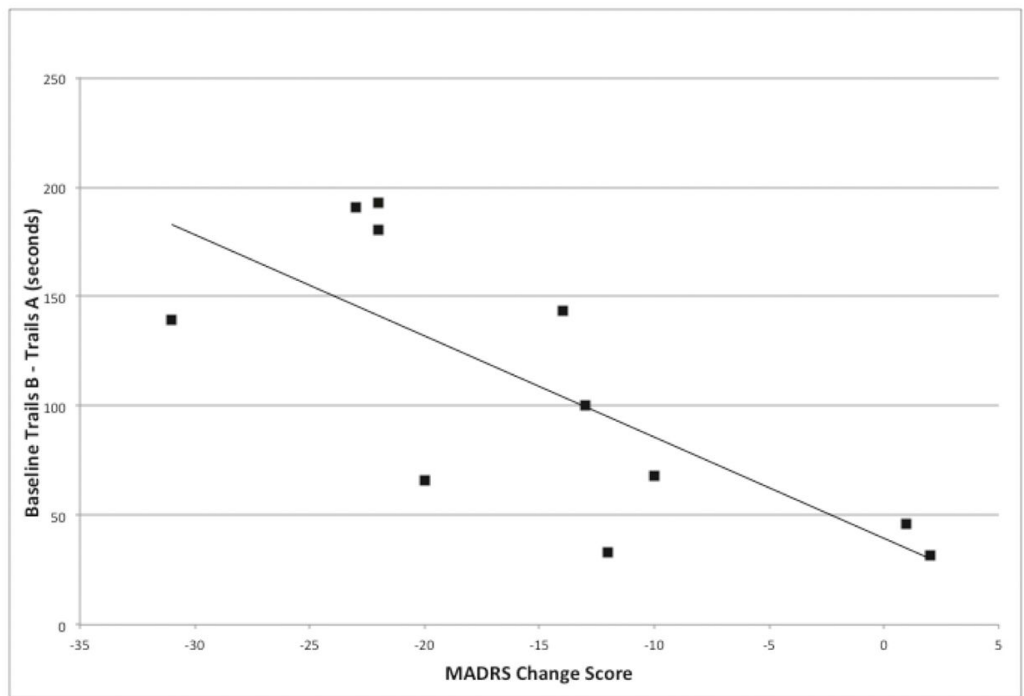

Figure 1.

Baseline Trails B (minus Trails A) performance (in seconds) in 11 elderly depressed patients by MADRS change scores after 4-weeks of treatment with nCCR-GD. 